\title{
Enfoques del facultamiento en la Dirección de Impuestos y Aduanas Nacionales (DIAN) de Santa Marta en Colombia
}

DOI: https://doi.org/10.21158/01208160.n83.2017.1820

Fecha de recepción: 25 de junio de 2017

Fecha de aprobación: 28 de septiembre de 2017

\author{
Zoraima Aurelia Donawa Torres ${ }^{1}$ \\ Instituto Universitario de Tecnología de Cabimas \\ zdonawa62@hotmail.com \\ Moreibis Irina López Redondo 2 \\ Universidad Dr. Rafael Belloso Chacín \\ mlopezr@dian.gov.co \\ Gladys Esther Vega Alfaro ${ }^{3}$ \\ Universidad Dr. Rafael Belloso Chacín \\ gladysevegaa@gmail.com
}

Cómo citar este artículo / To reference this article / Comment citer cet article / Para citar este artigo:

Donawa Torres, Z. A., López Redondo, M. I. y Vega Alfaro, G. E. (2017). Enfoques del facultamiento en la Dirección de Impuestos y Aduanas Nacionales de Santa Marta en Colombia. Revista EAN, 83, pp 167-178. https://doi.org/10.21158/01208160. n83.2017.1820

\section{Resumen}

Este artículo es el producto de la investigación cuyo propósito fundamental fue identificar los enfoques del facultamiento en la Dirección de Impuestos y Aduanas Nacionales de Santa Marta en Colombia. La metodología utilizada fue bajo el paradigma de investigación positivista, el tipo de investigación descriptiva, el diseño no experimental, transeccional y de campo. La población estuvo constituida por 188 empleados de la Dirección de Impuestos y Aduanas Nacionales de Santa Marta en Colombia. Como instrumento para la recolección de datos, se elaboró un cuestionario utilizando las categorías de respuestas de tipo Likert de 15 ítems, validado por expertos en el área. Los resultados de esta investigación, permiten evidenciar casi la ausencia total de los enfoques del empoderamiento como herramienta gerencial. Las conclusiones revelan que los empleados no se sienten competentes ni apreciados, consideran que su trabajo no tiene sentido y efecto, y que no tienen oportunidad de usar su talento.

\section{Palabras clave}

Facultamiento, enfoques de facultamiento, empoderamiento, empleados sector público.

${ }^{1}$ Politóloga, magíster en Gerencia de empresas. Doctora en Ciencias Gerenciales, con postdoctorado en Gerencia de las Organizaciones. Docente agregado e investigadora del Instituto Universitario de Tecnología de Cabimas de Venezuela.

${ }^{2}$ Especialista en Administración y Gerencia Institucional, doctorante en Ciencias Gerenciales. Gestor IV G.I.T Auditoria Tributaria I. DIAN. Colombia.

${ }^{3}$ Especialista en Tributación, doctorante en Ciencias Gerenciales. Revisor de la Contraloría General de la República (19882009). Jefe de la división de recaudo y cobranza. 


\title{
Different approaches to the entitlement of the National Directorate of Taxes and Customs (DIAN) in Santa Marta, Colombia
}

\begin{abstract}
This article results from a research study whose main purpose is to identify different approaches to the entitlement of the National Directorate of Taxes and Customs in Santa Marta, Colombia. The methodology used was the paradigm of positivist research, a type of descriptive research, non-experimental design, transectional and field work. The population was composed by 188 workers from the National Directorate of Taxes and Customs in Santa Marta, Colombia. To collect valuable data, a questionnaire having different answer categories taken from Likert fifteen item type was validated by area experts. The outcomes of this research show an almost total lack of empowerment approaches as managerial tools. To conclude, employees show that they are neither competent nor appreciated, considering that their work has no sense or direct impact and that they are not allowed to use their talent.
\end{abstract}

Key words. Entitlement, entitlement approaches, empowerment, public sector empoyees.

\section{Approche en termes de compétences au sein de la Direction des Impôts et des Douanes Nationales (DIAN) de Santa Marta, Colombie}

Résumé. Cette étude a pour objectif d'identifier les approches en termes de compétences au sein de la Direction des Impôts et des Douanes Nationales (DIAN) de Santa Marta, Colombie. La méthodologie utilisée suit le paradigme de recherche positiviste, descriptif, de conception non expérimentale et empirique. L'échantillon objet d'étude se compose de 188 employés de la Direction des Impôts et des Douanes Nationales de Santa Marta, Colombie. La collecte des données est effectuée au moyen d'un questionnaire élaboré suivant les catégories de réponses de type Likert de 15 points, validé par des experts du secteur. Les résultats de l'étude permettent de mettre en évidence une absence quasi totale d'outils de gestion des compétences. Les conclusions de l'étude révèlent que les employés interrogés se sentent peu appréciés, peu compétents, qu'ils considèrent que leur travail n'a pas de sens et qu'ils n'ont pas l'opportunité d'utiliser leurs talents.

Mots clefs. Compétences, approche par compétences, transfert de pouvoir, employés du secteur public.

\section{Enfoques do empoderamento na Direção Nacional de Impostos e Aduanas (DIAN) de Santa Marta na Colômbia}

Resumo. Este artigo é o produto da pesquisa cujo objetivo fundamental foi identificar os enfoques do empoderamento na Direção de Impostos Nacionais e Aduanas de Santa Marta, na Colômbia. A metodologia utilizada esteve sob o paradigma da pesquisa positivista, o tipo de pesquisa foi descritivo, desenho não-experimental, transversal e de campo. A população foi constituída por 188 funcionários da Direção Nacional de Impostos e Aduanas de Santa Marta, na Colômbia. Como instrumento de coleta de dados, aplicou-se um questionário usando as categorias de resposta do tipo Likert de 15 itens, validadas por especialistas na área. Os resultados desta pesquisa permitem demonstrar a ausência quase total de enfoques de empoderamento como ferramenta de gerenciamento. Os resultados revelam que os funcionários não se sentem competentes ou apreciados, consideram que seu trabalho não tem sentido e efeito e que eles não têm oportunidade de usar seu talento.

Palavras-chave. Empoderamento, enfoques de empoderamento, funcionários do setor público. 


\section{Introducción}

Z1 empoderamiento es una estrategia gerencial que fortalece la formación del talento humano en las organizaciones, pues constituye una herramienta para el mejor desarrollo de las habilidades profesionales de los empleados, genera una capacidad de decisión que antes no era posible, y crea una nueva forma de asumir las responsabilidades diarias que en algunos casos se considera agradable.

El empoderamiento consiste en capacitar y facultar para dar poder y autoridad a los empleados a fin de que tengan influencia y poder de decisión sobresu trabajo sin necesidad de requerir constante permiso para actuar, lo cual no significa una pérdida de autoridad de la alta gerencia, sino la generación en los trabajadores de una sensación de pertenencia que trae implícito el desarrollo del espíritu de colaboración empresa trabajador.

El objetivo de este artículo es identificar los enfoques del empoderamiento en la Dirección de Impuestos y Aduanas Nacionales de Santa Marta en Colombia. En este sentido, cada enfoque representa un objetivo específico. Cabe destacar, que los enfoques del empoderamiento, facultamiento, empoderamiento o empowerment, según autores, como Fred (2013), Blanchard (2007) Rabouin (2008) entre otros, son aplicados por la gerencia de las organizaciones como estrategia para que los empleados comiencen a crecer, sean competentes y apreciados, que su trabajo tenga sentido y efecto, y que tienen oportunidad de usar su talento.

\section{Facultamiento}

T os gerentes de las organizaciones deben Lmotivar a los empleados para que trabajen en función del logro de los elementos constitutivos de una ventaja competitiva a través de la estructura y la cultura organizacional. En este sentido, Hill y Jones (2009) señalan que el propósito de la estructura organizacional es coordinar e integrar los esfuerzos de los empleados de todos los niveles, corporativo, de negocios y funcional, puestos y unidades de negocios de una compañía, de manera que operen juntos en una forma que permita llevar a cabo la serie específica de estrategias en su modelo de negocios.
Esto significa que la estructura organizacional es la forma en la que los empleados de una empresa son distribuidos en función de los puestos de trabajo, sin embargo, la estructura organizacional es eficaz cuando facilita a las personas la consecución de los objetivos y es eficiente cuando se consiguen con recursos o costos mínimos (Chiavenato, 2009a).

En cuanto a la cultura organizacional, Serna (2003) revela que es la manera como las organizaciones hacen las cosas, establecen prioridades y dan importancia a las tareas. Es decir, son modos de hacer, pensar, creer, 
basados en valores y que establecen patrones de comportamiento en un grupo de personas y en un momento determinado (Pérez, Bonelli y Ayala, 2011). Así mismo, la cultura organizacional brinda una sensación de identidad a los miembros y aumenta su compromiso con la organización. Esto quiere decir que los empleados encuentran su trabajo satisfactorio cuando interiorizan los valores de la compañía y se identifican con sus compañeros (Nelson y Quick, 2013).

Dentro de este contexto, Siliceo, Casares y González (1999) advierten que la cultura laboral de las organizaciones para el siglo XXI estará fincada en líderes constructores del ambiente, en el que se den a plenitud los procesos humanos, orientados a procesos de mejora continua, que involucran el empowerment, pues comprenden la delegación, la automotivación, el desarrollo integral y la creatividad del personal a todos los niveles. Esto significa la búsqueda de estrategias y modelos de mayor participación del personal en los procesos de creatividad, innovación y toma de decisiones.

En el mismo orden de ideas, es conveniente acotar que este proceso humano utilizado como estrategia gerencial es denominado por algunos autores empowerment y por otros facultamiento o empoderamiento; no obstante, en este artículo, los tres nombres tienen el mismo significado, y se tratará, en lo sucesivo, de ofrecer los conceptos que expresan algunos autores.

Koontz y Weihrich (2007) acotan que empowerment es un término en inglés que quiere decir potenciación o empoderamiento, el cual se traduce al hecho de delegar poder y autoridad a los subordinados y de conferirles el sentimiento de ser dueños de su propio trabajo.
Bateman y Snell (2009) comentan que el término empowerment se utiliza con frecuencia al hablar de autoridad descentralizada, es decir, la gerencia debe otorgar autoridad a directivos de nivel inferior para que tomen decisiones que beneficien a la organización. En este sentido, definen el empowerment como el proceso de compartir poder con los empleados y así reforzar su confianza en su capacidad para desempeñar su trabajo y su creencia en que son colaboradores que influyen en la organización.

Por su parte, Alles (2007) señala que el término empoderamiento se puede entender como un método que tiene el objetivo de acercar lo más posible la toma de decisiones al lugar y momento donde ocurren los hechos. Así mismo, que el término empowerment significa habilitar, conceder, permitir una iniciativa a cierta persona para actuar por sí misma y permitir la iniciativa a otros.

Se puede entender que el empowerment es un sinónimo de cultura de participación donde se requiere el concurso de todos para alcanzar objetivos de calidad, donde las organizaciones rompen esquemas y paradigmas en cuanto a las decisiones, los estilos de liderazgo, el equilibrio en la utilidad beneficio dentro del eje patrón trabajador, la planeación de vida y carrera del personal, y sobre todo, en los procesos de comunicación e integración de grupos humanos de trabajo en torno a los valores y la cultura organizacional. En este sentido, los autores Conger y Kanungo (1988) proponen que el empowerment debe ser considerado un elemento provocador para estimular a los trabajadores, más que una sencilla delegación de poder, entendiendo que habilitar implica crear las condiciones que permitan aumentar la motivación para la ejecución de las tareas mediante el desarrollo de un fuerte sentido de eficacia personal. 
El empowerment implica disminución de mandos medios y renuncia de autoridad de estos, se define como una herramienta administrativa que permite analizar las estructuras de autoridad y división del trabajo, con el fin de incrementar las facultades de los colaboradores, unidades y equipos de trabajo para agilizar los procesos productivos y la toma de decisiones y disminuir los costos de nómina en una empresa (Hernández y Rodríguez, 2006).

Por su parte, Daft (2011) enuncia que el empowerment significa compartir el poder, la delegación del poder o la autoridad en los subordinados en una organización. Así mismo, afirma, que al incrementar el poder de los empleados, se aumenta la motivación para el logro de las tareas, debido a que las personas mejoran su efectividad, al decidir cómo desempeñar una tarea utilizando su creatividad. Un gerente que sepa delegar autoridad debe estar dispuesto a entregar el derecho de tomar decisiones a los subordinados. También requiere que los empleados y los equipos acepten la responsabilidad por sus acciones y su trabajo (Koontz y Weihrich, 2007).

Cabe destacar que Chiavenato (2009a) considera que el facultamiento en la toma de decisiones -empowerment- o delegación de autoridad parte de la idea de otorgar a las personas el poder, la libertad y la información que necesitan para tomar decisiones y participar activamente en la organización. Expresa, además, que en un entorno de negocios que se caracteriza por la intensa competencia global y el rápido surgimiento de nuevas tecnologías, soltar la rienda del control centralizado parece ser una solución viable que promueve la velocidad, la flexibilidad y la capacidad de decisión de la organización.
Este autor revela que el facultamiento en la toma de decisiones se funda en cuatro bases:

- Poder: otorgar poder a las personas mediante la delegación de autoridad y responsabilidades en todos los niveles de la organización. Esto significa dar importancia a las personas, confiar en ellas, otorgarles libertad y autonomía de acción.

- Motivación: motivar e incentivar a las personas continuamente significa reconocer el buen desempeño, recompensar los resultados, permitir que las personas participen de los resultados de su trabajo y festejar el logro de metas.

- Desarrollo: proporcionar recursos para facilitar la capacitación y el desarrollo personal y profesional significa adiestrar a las personas continuamente, ofrecerles información y conocimientos, enseñarles nuevas técnicas y crear y desarrollar talentos en la organización.

- Liderazgo: propiciar el liderazgo en la organización significa orientar a las personas, definir objetivos y metas, ampliar horizontes, evaluar el desempeño y ofrecer realimentación.

Es importante acotar que Whetten y Cameron (2005) hacen una distinción al decir que el poder y el facultamiento son diferentes:

- La fuente de poder es externa, mientras que la fuente de facultamiento es interna.

- El poder es la capacidad del jefe de hacer que los empleados hagan lo que él quiere; al contrario, el facultamiento es la capacidad de hacer que los empleados hagan lo que tienen que hacer. 
- Obtener más implica quitárselo a otra persona; en cambio, en el facultamiento obtener más no afecta cuánto tengan los demás.

- Pocas personas tienen el poder, pero en el facultamiento todos pueden tenerlo.

- El poder provoca competencia, mientras que el facultamiento lleva a la cooperación.

Cabe destacar que Conger y Kanungo (1988) advierten que, al incrementar el poder de los empleados, se aumenta la motivación para el logro de las tareas, debido a que las personas mejoran su efectividad al decidir cómo desempeñar una tarea utilizando su creatividad. Del mismo modo, los empleados perciben potencia; al sentirse competentes y capaces de lograr sus tareas, sienten autonomía al seleccionar la forma de cómo realizar sus tareas, y con un sentido de impacto, al experimentar la importancia de su trabajo realizado y las metas alcanzadas (Hellriegell, Jackson y Slocum, 2009).

En este mismo orden y dirección, desde el enfoque del talento humano, expresan Soto y Cárdenas (2007) que el llamado empoderamiento que se define como el uso consiente y deliberado de las posibilidades del ser humano dirigido hacia una meta o logro es la capacidad individual de diseñar el presente. El empoderamiento quiere decir que una persona usa sus recursos disponibles, como su cuerpo, juicios y emociones, a fin de actuar en forma coherente para lograr mayor potencia en sus acciones y efectividad en sus resultados.

El empoderamiento o adquisición de poder se puede ejercer a través de la expansión de las capacidades de aprendizaje, donde la persona, guiada por un experto, se conecta mediante sus recursos personales disponibles, imagina nuevas posibilidades de acción y actúa en la dirección que la llevará a lograrlo. De igual manera, Fred (2013) define el empoderamiento como el acto de fortalecer el sentimiento de eficacia en los empleados, el cual los alienta a participar en la toma de decisiones y a ejercer su iniciativa e imaginación, y se les recompensa por hacerlo.

Por su parte, Wilson (2004), refiere que el empoderamiento o empowerment, como estrategia gerencial, es un proceso iniciado por los gerentes que captan las ideas y los deseos de todos los empleados de la empresa permitiéndose que se desarrolle la totalidad de sus talentos y habilidades para la consecución de las metas de trabajo y el desarrollo profesional. Así mismo, precisa el autor, el empoderamiento consiste en potenciar la motivación y los resultados de todos los colaboradores de una empresa a través de la delegación y la transmisión del poder.

Blanchard (2007) define el facultamiento como la creación de un clima organizacional que libera el conocimiento, la experiencia y la motivación que residen en las personas. En este sentido, Blanchard y Randolph (1996), citados en Blanchard (2007), explican que los gerentes que dirigen una organización hacia una cultura de facultamiento deben utilizar tres claves: i) compartir información, ii) establecer las fronteras; y iii) reemplazar la antigua jerarquía por individuos y equipos autodirigidos. En otras palabras, el empowerment consiste en la liberación de los conocimientos, la experiencia y la capacidad de motivarse que tienen las personas (Rabouin, 2008). 


\section{Enfoques del facultamiento}

$\mathbf{E}_{\text {mayor autonomía a los empleados, que }}^{1 \text { facultamiento es un proceso que ofrece }}$ comparte con ellos información relevante y les da control sobre los factores que influyen en su desempeño laboral (Newstrom, 2011). Explica, además, que el facultamiento, en primer lugar, elimina las condiciones que causan impotencia, a la vez que refuerza los sentimientos de eficacia personal del empleado; y en segundo lugar, autoriza a los empleados a lidiar con las situaciones y les permite tomar el control de los problemas a medida que se presentan.
Este autor revela unos criterios o enfoques sobre el facultamiento señalando que cuando los gerentes los aplican los empleados comienzan a creer que son competentes y apreciados, que su trabajo tiene sentido y efecto y que tienen oportunidad de usar su talento, pues, cuando se les faculta legítimamente, es más probable que sus esfuerzos generen tanto satisfacción personal como la clase de resultados que la organización valora. No obstante, los describe como una cadena de sucesos que se reflejan a continuación (Figura 1).

Figura 1. Enfoques del facultamiento.

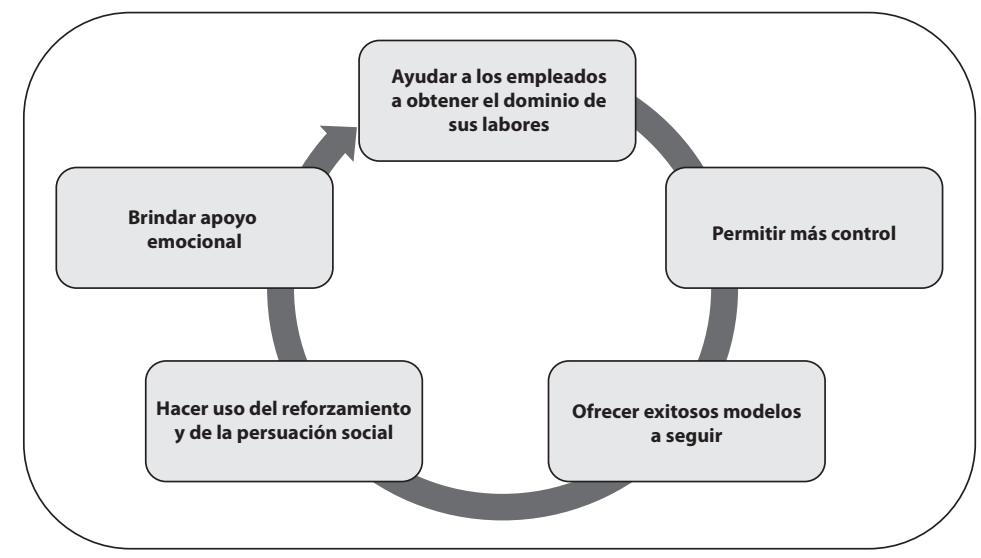

Fuente. Elaboración propia.

\subsection{Ayudar a los empleados a obtener el dominio de sus labores}

Newstrom (2011) acota, que al ayudar a los empleados a obtener el dominio de sus labores, ponen a su alcance una capacitación apropiada, el entrenamiento y la experiencia guiada, cuyo resultado serán éxitos iniciales. Así mismo, Siliceo, Casares y González (1999) enuncian que la capacitación consiste en una actividad planeada y basada en necesidades reales de una empresa u organización y orientada hacia un cambio en los conocimientos, las habilidades y las actitudes del colaborador.

La capacitación ayuda a los empleados a desempeñar su trabajo actual y sus beneficios pueden extenderse a toda la vida laboral o profesional de la persona y ayudar a desarrollarla para responsabilidades futuras (Siliceo, 2004). En este sentido, Arnold y Randall (2012) afirman, que en la actualidad, muchas organizaciones están empezando a ver la capacitación como un proceso continuo de aprendizaje organizacional que requiere que los empleados compartan conocimientos y aprendizaje entre sí. 


\subsection{Permitir más control}

Robbins y DeCenzo (2002) definen el control dentro de una organización como el proceso que consiste en vigilar las actividades, con el fin de asegurar que se realicen conforme a los planes y corregir las desviaciones importantes, pues un sistema de control garantiza que las actividades se cumplan de tal forma que se alcancen las metas de la organización.

No obstante, Newstrom (2011) considera que permitir más control hace que la gerencia esté concediendo a los empleados discrecionalidad sobre su desempeño laboral y responsabilizándolos de los resultados. En este sentido, el gerente proporciona una serie de incentivos a fin de motivar a los empleados para que trabajen con el fin de lograr una creciente eficiencia, calidad, innovación y actitud sensible ante los clientes, y una retroalimentación específica acerca del nivel de eficacia con que una organización y sus miembros se desempeñan y crean ventajas competitivas. El control personal es el que se deriva del contacto personal con los subordinados que se puede detectar en la supervisión directa de las acciones de los subordinados (Hill y Jones, 2009).

Por su parte, Chiavenato (2009b) acota que la evaluación de desempeño indica el valor del desempeño de cada persona, en función de las actividades que cumple, de las metas y los resultados que debe alcanzar y de su posible desarrollo; es un proceso que sirve para juzgar y estimar la excelencia de las cualidades de una persona, y sobre todo, su contribución al negocio de la organización.

\subsection{Ofrecer exitosos modelos}

En palabras de Newstrom (2011), ofrecer exitosos modelos permite a los empleados que observen a compañeros que ya se desempeñan exitosamente en sus labores. Vale decir que no es lo mismo un grupo que un equipo. Un grupo son dos o más individuos que interactúan, son interdependientes o se reúnen para lograr objetivos particulares. Un grupo de trabajo es aquel que interactúa principalmente para compartir información y tomar decisiones que ayuden al desempeño de cada uno de sus miembros en su área de responsabilidad.

En este sentido, Robbins y Judge (2013) acotan que un equipo de trabajo genera una sinergia positiva gracias al esfuerzo coordinado, es decir, es un grupo donde los esfuerzos individuales dan como resultado un desempeño que es mayor que la suma de las aportaciones de cada individuo.

\subsection{Hacer uso del reforzamiento y de la persuasión social}

La persuasión social es una técnica que consiste en dar a conocer periódicamente a los empleados el nivel de resultados alcanzados y aquellos aspectos en los cuales se espera una mejoría de su parte. El empleado cuya evaluación se realiza periódica y sistemáticamente tiende a esmerarse, pues sabe que se le observa y califica, que su esfuerzo no pasa inadvertido y que la organización toma interés en su trabajo. El propósito es suministrar una retroalimentación adecuada para los empleados en cuanto a su desempeño y la oportunidad de desarrollarse en las actividades de la organización. Toda evaluación del desempeño debe tener su propósito y objetivos claramente definidos, es decir, su finalidad; de esta forma, los resultados obtenidos podrán ser útiles al desarrollo de la planificación organizacional 
y al consecuente logro de los objetivos de productividad previamente establecidos (Arias y Heredia, 2006).

Advierte Newstrom (2011) que para el empoderamiento existe la necesidad de hacer uso del reforzamiento y la persuasión social ofreciendo elogios, aliento $y$ retroalimentación verbal diseñada para fortalecer la seguridad de los empleados en sí mismos. Además, se debe proporcionar retroalimentación positiva y negativa, ya que en la evaluación se encontrarán, aparte de debilidades, aspectos positivos del desempeño del trabajador, los cuales se deben afianzar a través del refuerzo positivo; informándole al empleado las actividades ha llevado a cabo de manera adecuada obtendrá una visión global de su desenvolvimiento (Robbins y DeCenso, 2008).

\subsection{Brindar apoyo emocional}

Resalta Newstrom (2011) que para empoderar hay que brindar apoyo emocional a los empleados, puesto que mediante la reducción de la tensión y la angustia se obtiene una mejor definición de funciones ayudando en las tareas y a la atención honesta. Destacan, además, que el líder adoptando estos enfoques influirá en el subordinado de forma tal que se deberá sentir motivado para la realización de sus tareas, el empleado debe sentir que estas tienen sentido, y por tanto, verlo reflejado en el desempeño diario de sus actividades.
Del mismo modo, Robbins y Judge (2013) acotan que las condiciones psicosociales consisten en factores relativos a la organización del trabajo que son decisivos para la realización personal del trabajador. Los factores psicosociales como aquellas condiciones que se encuentran presentes en una situación laboral que están directamente relacionadas con la organización, el contenido del trabajo y la realización de la tarea, y que tienen capacidad para afectar tanto el bienestar o la salud -física, psíquica o social- del trabajador como el desarrollo del trabajo.

Como algunos ejemplos de brindar apoyo, se sugiere elogiar, alentar, expresar aprobación y reafirmar, fomentar actividades sociales informales para aumentar la cohesión, dar retroalimentación de manera regular y realizar ceremonias de reconocimiento (Whetten y Cameron, 2011).

Cuando los gerentes aplican estos enfoques, los empleados comienzan a creer que son competentes y apreciados, que su trabajo tiene sentido y efecto y que tienen oportunidad de usar su talento. En efecto, cuando se les empodera o faculta legítimamente, es más probable que sus esfuerzos generen tanto satisfacción personal como la clase de resultados que la organización valora (Newstrom, 2011). 


\section{Metodología}

T a metodología utilizada fue bajo el Lparadigma de investigación positivista, el tipo de investigación descriptiva, el diseño no experimental, transeccional y de campo. La población estuvo constituida por 188 empleados de la Dirección de Impuestos y Aduanas Nacionales de Santa Marta en Colombia. Como instrumento para la recolección de datos, se elaboró un cuestionario utilizando las categorías de respuestas de tipo Likert de 15 ítems, validado por expertos en el área.
La validez del instrumento se llevó a cabo bajo el juicio de siete expertos sobre la variable en estudio. La confiabilidad del instrumento fue determinada por el método alfa de Cronbach presentando $0.98 \%$ de confiabilidad. Posteriormente, fue aplicado a la población del estudio. Finalmente, se agrupan los datos haciendo uso de la estadística descriptiva, construyendo las tablas de frecuencia con sus respectivos gráficos y posterior análisis e interpretación de los datos obtenidos.

\section{Resultados}

$\mathrm{T}$ eniendo en consideración, que en las organizaciones con cultura de facultamiento, los gerentes adoptan un comportamiento intencional impulsando el poder, la autoridad y la responsabilidad hacia los empleados para que puedan obtener el alcance de sus objetivos, la presencia de los enfoques del facultamiento se hace necesaria Tabla 1. Enfoques del empoderamiento. para distinguir su aplicación como estrategia gerencial. En este sentido, al identificar los enfoques del empoderamiento en la gerencia de la Dirección de Impuestos y Aduanas Nacionales de Santa Marta en Colombia, los resultados se agrupan de acuerdo con la siguiente información (Tabla 1).

\begin{tabular}{|c|c|c|c|c|c|c|c|c|c|c|c|c|}
\hline \multirow[b]{2}{*}{$\begin{array}{c}\text { Categorias de } \\
\text { respuestas }\end{array}$} & \multicolumn{2}{|c|}{ Ayuda } & \multicolumn{2}{|c|}{ Permitir } & \multicolumn{2}{|c|}{ Ofrecer } & \multicolumn{2}{|c|}{ Hacer } & \multicolumn{2}{|c|}{ Brindar } & \multirow[b]{2}{*}{$\mathrm{Fi}$} & \multirow[b]{2}{*}{$\%$} \\
\hline & $\mathrm{Fi}$ & $\%$ & $\mathrm{Fi}$ & $\%$ & $\mathrm{Fi}$ & $\%$ & $\mathrm{Fi}$ & $\%$ & $\mathrm{Fi}$ & $\%$ & & \\
\hline Muy de acuerdo. & 0 & 0 & 0 & 0 & 0 & 0 & 0 & 0 & 0 & 0 & 0 & 0 \\
\hline De acuerdo. & 84 & 44 & 125 & 67 & 84 & 56 & 84 & 44 & 84 & 44 & 92 & 51 \\
\hline $\begin{array}{l}\text { Ni de acuerdo, ni en } \\
\text { desacuerdo. }\end{array}$ & 104 & 56 & 21 & 11 & 104 & 33 & 63 & 33 & 63 & 33 & 71 & 33 \\
\hline En desacuerdo. & 0 & 0 & 42 & 22 & 0 & 11 & 42 & 22 & 42 & 22 & 25 & 16 \\
\hline Muy en desacuerdo. & 0 & 0 & 0 & 0 & 0 & 0 & 0 & 0 & 0 & 0 & 0 & 0 \\
\hline Total. & 188 & 100 & 188 & 100 & 188 & 100 & 188 & 100 & 188 & 100 & 188 & 100 \\
\hline
\end{tabular}

Fuente. Elaboración propia. 
Los resultados reflejados (Tabla 1), revelan que en el indicador Ayudar a los empleados a obtener el dominio de sus labores, 55 de los encuestados que representan $29 \%$, y 24 de ellos que representan $13 \%$, respondieron en forma positiva a las afirmaciones contenidas en el cuestionario al seleccionar la categoría de respuesta Verdadero y Completamente verdadero, respectivamente; 24 de los sujetos encuestados que representan 13 $\%$ y 24 que representan $13 \%$, seleccionaron las categorías de respuestas negativas falso y completamente falso, respectivamente. No obstante, 63 de los sujetos encuestados que representan $33 \%$ mantienen una posición neutral al seleccionar la categoría de respuesta Ni falso, ni verdadero.

De igual manera, en lo que respecta al indicador Permitir más control, los resultados reflejan que 71 de los sujetos encuestados que representan $38 \%$, y 24 de ellos que representan $13 \%$, respondieron en forma positiva a las afirmaciones contenidas en el cuestionario al seleccionar la categoría de respuesta verdadero y completamente verdadero, respectivamente; 39 de los encuestados que representan $21 \%$ y 24 que representan 13 $\%$, seleccionaron las categorías de respuestas negativas falso y completamente falso, respectivamente. No obstante, 31 de los encuestados que representan $17 \%$ mantienen una posición neutral al seleccionar la categoría de respuesta ni falso, ni verdadero.

Vale decir que los resultados reflejados en el indicador ofrecer exitosos modelos permiten evidenciar que 55 de los sujetos encuestados que representan $33 \%$, y 24 de ellos que representan $13 \%$, respondieron en forma positiva a las afirmaciones contenidas en el cuestionario al seleccionar la categoría de respuesta verdadero y completamente verdadero, respectivamente; 24 de los sujetos encuestados que representan 17 $\%$, y 24 que representan $13 \%$, seleccionaron las categorías de respuestas negativas falso y completamente falso, respectivamente. No obstante, 63 de los sujetos encuestados que representan $25 \%$ mantienen una posición neutral al seleccionar la categoría de respuesta ni falso, ni verdadero.

En cuanto al indicador hacer uso del reforzamiento y la persuasión social, los resultados arrojados en la investigación develan que 55 de los sujetos encuestados que representan 29 $\%$ y 24 de ellos que representan $13 \%$ respondieron en forma positiva a las afirmaciones contenidas en el cuestionario al seleccionar la categoría de respuesta verdadero y completamente verdadero, respectivamente; 39 de los sujetos encuestados que representan 21 $\%$ y 24 que representan $13 \%$ seleccionaron las categorías de respuestas negativas falso y completamente falso, respectivamente. No obstante, 47 de los sujetos encuestados que representan $25 \%$ mantienen una posición neutral al seleccionar la categoría de respuesta ni falso, ni verdadero.

Finalmente, es de observar, que en el indicador brindar apoyo emocional, los resultados arrojan que 55 de los sujetos encuestados que representan $29 \%$, y 24 de ellos que representan $1 \%$ respondieron en forma positiva a las afirmaciones contenidas en el cuestionario al seleccionar la categoría de respuesta verdadero y completamente verdadero, respectivamente; 39 de los sujetos encuestados que representan 21 $\%$ y 24 que representan $13 \%$, seleccionaron las categorías de respuestas negativas falso y completamente falso, respectivamente. No obstante, 47 de los sujetos encuestados que representan $25 \%$ mantienen una posición neutral al seleccionar la categoría de respuesta ni falso, ni verdadero. 
Tal como se ilustra (Figura 2), al sumar las categorías de respuestas positivas, se puede observar que 79 de los empleados que representan $42 \%$ responden positivamente sobre la preocupación de la gerencia en ayudar a los empleados a obtener el dominio de sus labores; 95 de los empleados que representan $51 \%$ consideran cierto que la gerencia propicia permitir más control; 79 de los empleados que representan $46 \%$ percibe que la gerencia se preocupa por ofrecer exitosos modelos; 79 de los empleados que representan $42 \%$ consideran que la gerencia fomenta hacer uso del reforzamiento y la persuasión social; y finalmente, 79 de los empleados que representan $42 \%$ aceptan que la gerencia brinda apoyo emocional a los empleados.

Figura 2. Enfoques del facultamiento.

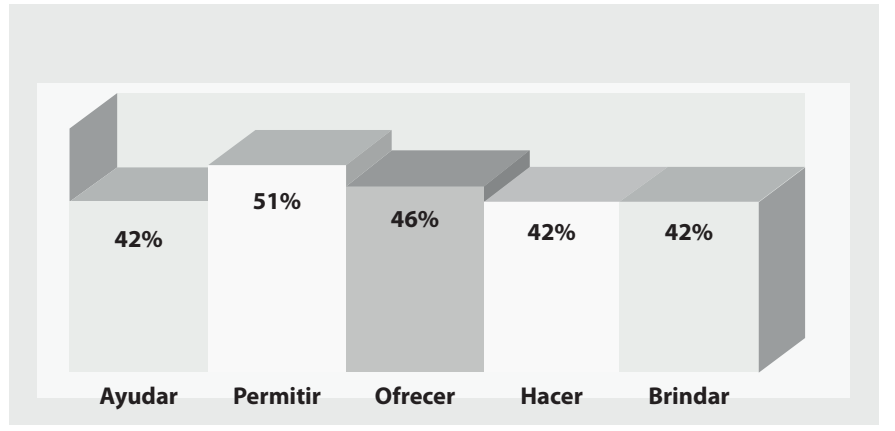

Fuente. Elaboración propia.

Tal como lo ilustra la figura 2, al sumar las categorías de respuestas positivas, se puede observar que 79 de los empleados que representan $42 \%$ responden positivamente sobre la preocupación de la gerencia en ayudar a los empleados a obtener el dominio de sus labores, es decir, para la mayoría de los empleados de la organización, la gerencia no contribuye a que los empleados puedan incrementar el dominio de sus actividades en función de su capacitación. Del mismo modo, los resultados evidencian que 95 de los empleados que representan $51 \%$ consideran cierto que la gerencia propicia permitir más control, lo cual significa que solo la mitad más uno de los empleados considera que la gerencia de la organización les concede discrecionalidad por su desempeño e incentivos que impulsen su motivación para trabajar con eficiencia y elevar su calidad laboral.
En el mismo orden de ideas, vale decir que 79 de los empleados que representan $46 \%$ percibe que la gerencia se preocupa por ofrecer exitosos modelos, por lo cual se evidencia que impide que los empleados puedan compartir información que les permita tomar decisiones para su mejor desempeño. Así mismo, 79 de los empleados que representan $42 \%$ consideran que la gerencia fomenta hacer uso del reforzamiento y la persuasión social, por lo cual se revela que para la mayoría de los empleados de la organización la gerencia no suministra adecuada retroalimentación por su desempeño a los empleados obstaculizando en ellos la seguridad en sí mismos.

Finalmente, vale decir que 79 de los empleados que representan $42 \%$ aceptan que la gerencia les brinda apoyo emocional, lo cual significa que la mayoría de los empleados de la organización considera un ambiente laboral tensionado y de angustia, con ausencia de motivación con actividades sociales saludables. 


\section{Conclusiones}

Tos resultados alcanzados sobre los Lenfoques del empoderamiento en la gerencia de las entidades públicas de Santa Marta en Colombia permiten inferir que la gerencia no ofrece mayor autonomía a los empleados compartiendo con ellos información relevante y dándoles control sobre los factores que influyen en su desempeño laboral, no suministra una retroalimentación adecuada para los empleados en cuanto a su desempeño ni la oportunidad de desarrollarse en las actividades de la organización. Esto significa que la gerencia no brinda el apoyo de los factores psicosociales como aquellas condiciones que se encuentran presentes en una situación laboral que están directamente relacionadas con la organización.

Del mismo modo, se infiere que los gerentes no aplican estos enfoques, por lo cual los empleados no creen ser competentes y apreciados, consideran que su trabajo no tiene sentido y efecto y que no tienen oportunidad de usar su talento. En efecto, cuando no se les faculta legítimamente, no sienten que sus esfuerzos generen satisfacción personal, pues no se sienten valorados por la organización.

\section{Referencias}

Alles, M. (2007). Comportamiento organizacional: cómo lograr un cambio cultural a través de gestión por competencias. Buenos Aires: Granica.

Arias Galicia, F. y Heredia Espinosa, V. (2006). Administración de recursos humanos para el alto desempeño (6. ${ }^{\mathrm{a}}$ ed.). México: Trillas.

Arnold, J. y Randall, R. (2012). Psicología del trabajo: comportamiento humano en el ámbito laboral (5. ${ }^{\mathrm{a}}$ ed.). México: Pearson.

Bateman, T. y Snell, S. (2009). Administración: liderazgo $y$ colaboración en un mundo competitivo (8. ${ }^{\mathrm{a}}$ ed.). México: McGraw-Hill.

Blanchard, K. (2007). Liderazgo al más alto nivel: cómo crear y dirigir organizaciones de alto desempeño. Bogotá: Norma.

Chiavenato, I. (2009a). Comportamiento organizacional: la dinámica del éxito en las organizaciones (2. ${ }^{\mathrm{a}}$ ed.). México: McGraw-Hill.

Chiavenato, I. (2009b). Gestión del talento humano (3. ed.). México: McGraw-Hill.
Conger, J. A. y Kanungo, R. N. (1988). The empowerment process: Integrating theory and practice. Academy of Management Review, 13(3), 471482.

Daft, R. (2011). Teoría y diseño organizacional (10. $\left.{ }^{\mathrm{a}} \mathrm{ed}.\right)$. México: Cengage.

Fred, D. (2013). Conceptos de administración estratégica (14. ${ }^{\mathrm{a}}$ ed.). México: Pearson.

Hellriegell, D., Jackson, S. y Slocum, J. (2009). Administración: un enfoque basado en competencias (11. ${ }^{\mathrm{a}}$ ed.). México: Cengage.

Hernández y Rodríguez, S. (2006). Introducción a la administración. Teoría general administrativa: origen, evolución y vanguardia (4. ${ }^{\mathrm{a}}$ ed.). México: McGrawHill.

Hill, C. y Jones, G. (2009). Administración estratégica (8. ${ }^{\mathrm{a}}$ ed.). México: McGraw-Hill.

Koontz, H. y Weihrich, H. (2007). Elementos de administración: un enfoque internacional (7. ${ }^{\mathrm{a}}$ ed.). México: McGraw-Hill. 
Nelson, D. y Quick, J. (2013). Comportamiento organizacional ( $3 .^{\mathrm{a}}$ ed.). México: Cengage.

Newstrom, J. (2011). Comportamiento humano en el trabajo (13. ${ }^{a}$ ed.). México: McGraw-Hill.

Pérez, L., Bonelli, M. y Ayala, J. (2011). El comportamiento de las personas en las organizaciones. Buenos Aires: Pearson Prentice-Hall.

Rabouin, R. (2008). Habilidades directivas para un nuevo management. Buenos Aires: Prentice Hall.

Robbins, S. y DeCenzo, D. (2002). Fundamentos de administración: conceptos esenciales y aplicaciones $\left(3{ }^{\mathrm{a}}\right.$ ed.). México: Pearson.

Robbins, S. y DeCenzo, D. (2008). Fundamentos de administración: conceptos esenciales y aplicaciones $\left(6 .^{\mathrm{a}}\right.$ ed.). México: Pearson.

Robbins, S. y Judge, T. (2013). Comportamiento organizacional (15. ${ }^{a}$ ed.). México: Pearson.

Serna Gómez, H. (2003). Gerencia estratégica: teoría, metodología, alineamiento, implementación y mapas estratégicos. Bogotá: 3R Editores.

Siliceo Aguilar, A. (2004). Capacitación y desarrollo de personal. México: Limusa.

Siliceo Aguilar, A., Casares Arrangoiz, D. y González Martínez, J. L. (1999). Liderazgo, valores y cultura organizacional: hacia una organización competitiva. México: McGraw-Hill.

Soto Pineda, E. y Cárdenas, J. A. (2007). Ética en las organizaciones. México: McGraw-Hill.

Whetten, D. y Cameron, K. (2005). Desarrollo de habilidades directivas (6. ${ }^{a}$ ed.). México: Pearson.

Whetten, D. y Cameron, K. (2011). Desarrollo de habilidades directivas (8. ${ }^{\mathrm{a}}$ ed.). México: Pearson.

Wilson, T. (2004). Manual del empowerment: cómo conseguir lo mejor de sus colaboradores. Barcelona: Gestión 2000. 